\title{
A Symplectic Lanczos-Type Algorithm to Compute the Eigenvalues of Positive Definite Hamiltonian Matrices
}

\author{
Pierluigi Amodio \\ Dipartimento di Matematica, Università di Bari, \\ Via E. Orabona 4, I-70125 Bari, Italy, \\ amodio@dm.uniba.it
}

\begin{abstract}
The Lanczos algorithm is a well known procedure to compute few eigenvalues of large symmetric matrices. We slightly modify this algorithm in order to obtain the eigenvalues of Hamiltonian matrices $H=$ $J S$ with $S$ symmetric and positive definite. These matrices represent a significant subclass of Hamiltonian matrices since their eigenvalues lie on the imaginary axis. An implicitly restarted procedure is also considered in order to speed-up the convergence of the algorithm.
\end{abstract}

\section{Introduction}

Many applications require the numerical approximation of the eigenvalues of a $2 n \times 2 n$ real Hamiltonian matrix $H=J S$ where

$$
J=\left(\begin{array}{cc}
O & I_{n} \\
-I_{n} & 0
\end{array}\right)
$$

and $S$ is a large and sparse symmetric matrix. As an example, we cite the solution of the continuous-time algebraic Riccati equations 911 of the form

$$
Q+A^{T} X+X A-X G X=0
$$

where $A, G$ and $Q$ are known $n \times n$ matrices, $G$ and $Q$ symmetric, and the solution $X$ is also symmetric.

It is well known that the considered eigenvalues are symmetric with respect to the real and imaginary axes. In this paper we are particularly interested in a subclass of Hamiltonian matrices where $S$ is symmetric and positive definite. In this case the eigenvalues lie (two by two symmetrically with respect to the origin) along the imaginary axis. These matrices arise in many application fields that deal with evolutionary problems whose solutions satisfy a certain conservation law, typically the energy of the system (see, for example, [5]). In the rest of the paper we refer to these matrices as positive definite Hamiltonian matrices [2].

All the numerical methods for the eigenvalues computation of Hamiltonian matrices use symplectic transformations in order to maintain the Hamiltonian

\footnotetext{
* Work supported by GNCS.
} 
structure of the matrix. In fact, if $P$ is symplectic (that is, $P^{T} J P=J$ ) and $H$ is Hamiltonian, then also $P^{-1} H P$ is Hamiltonian. For this reason, most of the existing algorithms are symplectic modifications of more general approaches as, for instance, the symplectic QR method in 6/7/3] and the symplectic iterative methods 3|4.

In particular, the symplectic Lanczos algorithm in 4] where the directions generated by the procedure are symplectic rather than orthogonal, is strictly related to the method introduced in this paper. The idea inherited this algorithm is the following: let

$$
S=\left(\begin{array}{cc}
S_{11} & S_{12} \\
S_{12}^{T} & S_{22}
\end{array}\right)
$$

with $S_{11}$ and $S_{22}$ symmetric, the matrix $H$ may be represented in the form

$$
H=\left(\begin{array}{cc}
S_{12}^{T} & S_{22} \\
-S_{11} & -S_{12}
\end{array}\right) \equiv\left(\begin{array}{cc}
V & W \\
Z & -V^{T}
\end{array}\right) .
$$

The approach in [4, as well as those in [1,10] which define canonical forms for the Hamiltonian matrices, transform the above matrix in

$$
\left(\begin{array}{cc}
V_{1} & W_{1} \\
& -V_{1}^{T}
\end{array}\right)
$$

with $V_{1}$ triangular and $V_{1}$ and $W_{1}$ having few nonnull diagonals. This is called a Hamiltonian triangular form for the matrix $H$.

Conversely, based on a theoretical result in [2, for positive definite Hamiltonian matrices we derive a canonical form ( $W_{2}$ tridiagonal)

$$
\left(\begin{array}{l}
W_{2} \\
-I
\end{array}\right)
$$

by means of a transformation that we prove to be symplectic. We will explain how to derive this form in Section 2. Then in Section 3, following the results presented in [48], we derive an implicit restarting technique that can be easily applied to the considered class of matrices.

\section{Tridiagonal Canonical Form}

In 2] existence results on a diagonal form for Hamiltonian matrices $H=J S$ with $S$ positive definite is given. In the same paper, this result is also rearranged in order to obtain the following symplectic transformation in the simplest canonical form.

Theorem 1. Given a real Hamiltonian matrix $H=J S$ with $S$ symmetric and positive definite, a real symplectic matrix $Z$ exists such that

$$
H Z=Z\left(\begin{array}{cc} 
& D_{1} \\
-D_{1} &
\end{array}\right) \equiv Z J\left(\begin{array}{ll}
D_{1} & \\
& D_{1}
\end{array}\right)
$$

with $D_{1}>0$ diagonal. 
Even if the proof of Theorem 1 is presented in [2], it is interesting to sketch its main steps. As a remark, we recall that:

- if $A$ is a symmetric matrix, then all the eigenvalues are real and an orthogonal matrix $U$ exists such that

$$
A U=U \Lambda
$$

- if $A$ is a skew-symmetric matrix, then all the eigenvalues are pure imaginary and a unitary matrix $U=\left[U_{1} \bar{U}_{1}\right]$ exists such that

$$
A U=i U\left(\begin{array}{ll}
\Lambda_{1} & \\
& -\Lambda_{1}
\end{array}\right)
$$

Moreover, from $V=\sqrt{2}\left[\operatorname{Re}\left(U_{1}\right) \operatorname{Im}\left(U_{1}\right)\right]$ one has

$$
A V=V J\left(\begin{array}{ll}
\Lambda_{1} & \\
& \Lambda_{1}
\end{array}\right), \quad V^{T} V=I,
$$

that is, a skew-symmetric matrix is similar to a positive definite Hamiltonian matrix by means of an orthogonal matrix.

Proof. Let us start from the decomposition of the symmetric and positive definite matrix $S$ in diagonal form, that is $S=Q \Lambda Q^{T}$, with $Q$ orthogonal and $\Lambda$ positive. Then

$$
(J S) J Q=\left(J Q \Lambda Q^{T}\right) J Q=J Q\left(\Lambda Q^{T} J Q\right)
$$

shows that $J S$ is similar to $\Lambda Q^{T} J Q$ by means of the transformation matrix $J Q$. Now, since the matrix $\Lambda^{1 / 2} Q^{T} J Q \Lambda^{1 / 2}$ is skew-symmetric, it will admit the following similarity transformation

$$
\Lambda^{1 / 2} Q^{T} J Q \Lambda^{1 / 2}=V J\left(\begin{array}{cc}
D_{1} & \\
& D_{1}
\end{array}\right) V^{T}
$$

and hence

$$
\Lambda Q^{T} J Q=\Lambda^{1 / 2}\left(\Lambda^{1 / 2} Q^{T} J Q \Lambda^{1 / 2}\right) \Lambda^{-1 / 2}=\left(\Lambda^{1 / 2} V\right) J\left(\begin{array}{cc}
D_{1} & \\
& D_{1}
\end{array}\right)\left(\Lambda^{1 / 2} V\right)^{-1} .
$$

Let $U=J Q \Lambda^{1 / 2} V$. One has that

$$
\begin{aligned}
U^{T} J U & =-\left(V^{T} \Lambda^{1 / 2} Q^{T} J\right) J\left(J Q \Lambda^{1 / 2} V\right)=V^{T} \Lambda^{1 / 2} Q^{T} J Q \Lambda^{1 / 2} V \\
& =V^{T} V J\left(\begin{array}{cc}
D_{1} & \\
& D_{1}
\end{array}\right) V^{T} V=J\left(\begin{array}{cc}
D_{1} & \\
& D_{1}
\end{array}\right)
\end{aligned}
$$

and therefore the matrix

$$
Z=U\left(\begin{array}{ll}
D_{1}^{-1 / 2} & \\
& D_{1}^{-1 / 2}
\end{array}\right)=J Q \Lambda^{1 / 2} V\left(\begin{array}{ll}
D_{1}^{-1 / 2} & \\
& D_{1}^{-1 / 2}
\end{array}\right)
$$

is symplectic and satisfies (11). 
Equation (11) allows us to easily obtain the eigenvalues of $J S$ as $i \lambda_{j}$ and $-i \lambda_{j}$, where $\lambda_{j}$ is a diagonal element of $D_{1}$. On the other hand, as it occurs in the case of symmetric matrices, the above theorem cannot be used to compute the eigenvalues numerically. For this reason, different approaches need to be introduced.

We now observe that $-S^{1 / 2} J S J S^{1 / 2}$ is symmetric and positive definite, then it is possible to apply to this matrix the Lanczos algorithm, that is one should compute a matrix $Z_{k+1}=\left[Z_{k} z_{k+1}\right]$ which is defined by means of $k+1$ orthogonal columns and a tridiagonal matrix $T_{k}$ such that

$$
-S^{1 / 2} J S J S^{1 / 2} Z_{k}=Z_{k} T_{k}+\beta_{k+1} z_{k+1} e_{k}^{T},
$$

where $e_{k}$ is the last unit vector of $\mathbb{R}^{k}$. Then, from $V_{k+1}=S^{-1 / 2} Z_{k+1}=$ $\left[\begin{array}{ll}V_{k} & v_{k+1}\end{array}\right]$, one has

$$
-H^{2} V_{k}=V_{k} T_{k}+\beta_{k+1} v_{k+1} e_{k}^{T}
$$

with $V_{k}$ of size $2 n \times k$ and such that

$$
V_{k}^{T} S V_{k}=I_{k}
$$

If $-i \lambda_{j}$, for $j=1, \ldots, n$, are the eigenvalues of $H$, then the eigenvalues of $H^{2}$ are $-\lambda_{1}^{2},-\lambda_{1}^{2},-\lambda_{2}^{2},-\lambda_{2}^{2}, \ldots$, that is, $H^{2}$ has double eigenvalues with a subspace of size 2 associated. This means that, if no breakdown has previously occurred, this algorithm theoretically will stop after $n$ steps, providing a tridiagonal symmetric and positive definite matrix $T$. In fact, from

$$
-H^{2} V=V T, \quad V^{T} S V=I_{n},
$$

one has

$$
-V^{T} S H^{2} V=V^{T} S V T=T
$$

and $-S H^{2}$ is symmetric and positive definite.

Hence the eigenvalues of $\mathrm{H}^{2}$ may be obtained applying an algorithm for symmetric and positive definite matrices to a half sized matrix $T$. Unfortunately, the decomposition (3)-(4) is not stable and can be only used to compute a limited number of extremal eigenvalues. Otherwise, it needs continue reorthogonalizations which make necessary to store all the columns of $\mathrm{V}$.

Anyway, from a theoretical point of view, we derive the following symplectic transformation which is numerically more reliable than that defined in Theorem 1.

Theorem 2. Given a real Hamiltonian matrix $H=J S$ with $S$ symmetric and positive definite, a real symplectic matrix $U$ exists such that

$$
H U=U\left(\begin{array}{c}
T \\
-I
\end{array}\right) \equiv U J\left(\begin{array}{ll}
I & \\
& T
\end{array}\right)
$$

with $T$ symmetric and positive definite tridiagonal matrix. 
Proof. Let

$$
W=-H V
$$

from (5) one has

$$
H W=V T
$$

and, consequently,

$$
H\left[\begin{array}{ll}
V & W
\end{array}\right]=\left[\begin{array}{ll}
-W & V T
\end{array}\right]=\left[\begin{array}{ll}
V & W
\end{array}\right]\left(\begin{array}{c}
T \\
-I
\end{array}\right) .
$$

The matrix $U=[V W]$ is symplectic, that is

$$
U^{T} J U=\left(\begin{array}{cc}
V^{T} J V & V^{T} J W \\
W^{T} J V & W^{T} J W
\end{array}\right)=J
$$

In fact, from (6) one has $J W=S V$ and

$$
V^{T} J W=V^{T} S V=I .
$$

Moreover, $V^{T} J V=O$ since each column $v_{i}$ of $V$ may be expressed in terms of an even polynomial of degree $2(i-1)$ in $H$, that is $v_{i}=p_{i-1}\left(H^{2}\right) v_{1}$, and for any integers $i$ and $l$

$$
v_{i}^{T} J v_{l}=v_{1}^{T}\left(p_{i-1}\left(H^{2}\right)\right)^{T} J p_{l-1}\left(H^{2}\right) v_{1}=v_{1}^{T}\left(p_{i+l-1}(H)\right)^{T} J p_{i+l-1}(H) v_{1}=0
$$

being, for any vector $v$ of appropriate length, $v^{T} J v=0$. Finally, $W^{T} J W=O$ for a similar reasoning.

The above theorem states that $U$ transforms the matrix $H$ in a matrix

$$
J\left(\begin{array}{ll}
I & \\
& T
\end{array}\right)
$$

which corresponds to the tridiagonal form of symmetric matrices. This is the simplest canonical transformation which can be computed for Hamiltonian matrices and proves the existence of a canonical form for positive definite Hamiltonian matrices which is slightly different from the one obtained in Theorem 1 In fact, from $T=Q_{1} D_{1}^{2} Q_{1}^{T}$, with $D_{1}$ diagonal and $Q_{1}$ orthogonal, it results that $\operatorname{diag}\left(Q_{1}, Q_{1}\right)$ is symplectic and

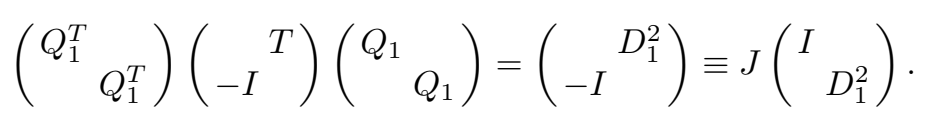

Now we derive an algorithm for the computation of matrices $U$ and $T$ in Theorem [2, Let $V_{k}=\left[v_{1}, \ldots, v_{k}\right]$ and 


$$
T_{k}=\left(\begin{array}{cccc}
\alpha_{1} & \beta_{2} & & \\
\beta_{2} & \alpha_{2} & \ddots & \\
& \ddots & \ddots & \beta_{k} \\
& & \beta_{k} & \alpha_{k}
\end{array}\right)
$$

the generic row of (3) is

$$
-H^{2} v_{j}=\beta_{j} v_{j-1}+\alpha_{j} v_{j}+\beta_{j+1} v_{j+1}
$$

and allows us to compute $v_{j+1}$. The coefficient $\beta_{j}$ is computed in order to have $v_{j}^{T} S v_{j}=1$, while $\alpha_{j}$ derives from

$$
0=v_{j}^{T} S v_{j+1}=\frac{1}{\beta_{j+1}}\left(v_{j}^{T} S H w_{j}-\alpha_{j}\right)=\frac{1}{\beta_{j+1}}\left(-v_{j}^{T} H^{T} S w_{j}-\alpha_{j}\right)
$$

where $w_{j}=-H v_{j}$ in analogy with (6)).

The complete algorithm to obtain the tridiagonal form is the following:

$$
\begin{aligned}
& \tilde{v}_{1} \text { arbitrary } \\
& \beta_{1}=\left(\tilde{v}_{1}^{T} S \tilde{v}_{1}\right)^{1 / 2} \\
& \text { for } j=1,2, \ldots, n \\
& \quad v_{j}=\tilde{v}_{j} / \beta_{j} \\
& w_{j}=-H v_{j} \\
& \quad \alpha_{j}=w_{j}^{T} S w_{j} \\
& \text { if } j=1 \\
& \quad \tilde{v}_{j+1}=H w_{j}-\alpha_{j} v_{j} \\
& \text { elseif } j<n \\
& \quad \tilde{v}_{j+1}=H w_{j}-\alpha_{j} v_{j}-\beta_{j} v_{j-1} \\
& \quad \text { end } \\
& \quad \beta_{j+1}=\left(\tilde{v}_{j+1}^{T} S \tilde{v}_{j+1}\right)^{1 / 2} \\
& \text { if } \beta_{j+1}=0, \text { stop } \\
& \text { end }
\end{aligned}
$$

We observe that $\alpha_{j}>0$ and $\beta_{j} \geq 0$. The algorithm stops before $n$ steps if $\beta_{j}=0$ (i.e., $\tilde{v}_{j}=0$ ), that is, when $\tilde{v}_{1}$ belongs to an invariant subspace of $H^{2}$. The vectors $w_{j}$ need not to be stored, while the $v_{j}$ are required for any reorthogonalization. If the above algorithm is applied to matrices $J S$ with $S$ nondefinite, then $\tilde{v}_{j}^{T} S \tilde{v}_{j}$ should be less than zero and the procedure breaks. A modification of the algorithm in order to overcome this problem will be investigated in future.

\section{Implicit Restarting}

As observed previously, the Lanczos method is widely used to compute only a small subset of the eigenvalues. In this case, the algorithm is stopped after a fixed 
number $k$ of iterations, then it restarts with a different initial vector $v_{1}$ obtained by the performed iterations. The idea is just to compute the new starting vector in order to obtain, after $k$ steps, $\beta_{k+1}=0$, that is an invariant subspace which allows us to compute $k$ eigenvalues.

The implicit restarted Lanczos algorithm shows several advantages since the storage requirement is fixed, there are no spurious eigenvalues, and deflation techniques similar to those applied to the QR algorithm may be applied [8].

As an example, we now analyze how it is possible to apply this technique to the Lanczos algorithm described in the previous section. Let us start from $k+2$ steps of equation (3)

$$
-H^{2} V_{k+1}=V_{k+1} T_{k+1}+\beta_{k+2} v_{k+2} e_{k+1}^{T}
$$

where $e_{k+1}$ represents the last unit vector of $\mathbb{R}^{k+1}$. Then, for a given real parameter $\mu$ and from $T_{k+1}-\mu I=Q R$ we have the following equalities

$$
\begin{aligned}
\left(-H^{2}-\mu I\right) V_{k+1} & =V_{k+1}\left(T_{k+1}-\mu I\right)+\beta_{k+2} v_{k+2} e_{k+1}^{T} \\
\left(-H^{2}-\mu I\right) V_{k+1} & =V_{k+1} Q R+\beta_{k+2} v_{k+2} e_{k+1}^{T} \\
\left(-H^{2}-\mu I\right)\left(V_{k+1} Q\right) & =\left(V_{k+1} Q\right) R Q+\beta_{k+2} v_{k+2} e_{k+1}^{T} Q \\
-H^{2}\left(V_{k+1} Q\right) & =\left(V_{k+1} Q\right)(R Q+\mu I)+\beta_{k+2} v_{k+2} e_{k+1}^{T} Q
\end{aligned}
$$

where $R Q+\mu I$ is a symmetric and positive definite tridiagonal matrix. The last equation cannot be however considered as obtained by a Lanczos procedure since

$$
\beta_{k+2} e_{k+1}^{T} Q=\left[\widehat{\beta}_{k+1} e_{k}^{T} \quad \widetilde{\beta}_{k+2}\right],
$$

that is, it has two elements different from zero (instead of one, see (99)). Anyway, equation (10) is useful to define the vector $v_{1}^{+}=\left(V_{k+1} Q\right) e_{1}\left(e_{1}\right.$ is the first unit vector of $\mathbb{R}^{k+1}$ ) as the starting vector of the new Lanczos iterations. Its relation with $v_{1}$ is

$$
\left(-H^{2}-\mu I\right) v_{1}=\rho_{1} v_{1}^{+}
$$

where $\rho_{1}$ is the $(1,1)$ element of $R$, is obtained by applying the second equation of (10) to $e_{1}$ and from $R e_{1}=\rho_{1} e_{1}$.

Let us now partition the matrices $V_{k+1} Q=\left[V_{k}^{+} \tilde{v}_{k+1}\right]$ and

$$
R Q+\mu I=Q^{T} T_{k+1} Q=\left(\begin{array}{cc}
T_{k}^{+} & \widetilde{\beta}_{k+1} e_{k} \\
\widetilde{\beta}_{k+1} e_{k}^{T} & \widetilde{\alpha}_{k+1}
\end{array}\right) .
$$

Substitution of (11) and (12) in the last equation of (10) gives

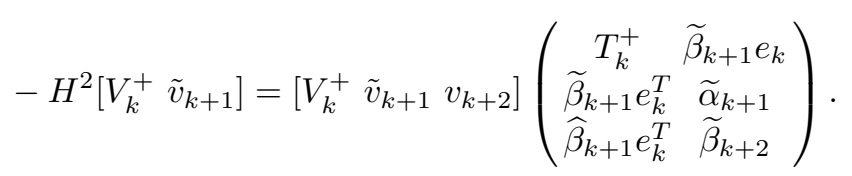

The first $k$ columns of (13) may be rewritten in the form 


$$
-H^{2} V_{k}^{+}=V_{k}^{+} T_{k}^{+}+\beta_{k+1}^{+} v_{k+1}^{+} e_{k}^{T}
$$

where

$$
v_{k+1}^{+}=\frac{1}{\beta_{k+1}^{+}}\left(\widetilde{\beta}_{k+1} \tilde{v}_{k+1}+\widehat{\beta}_{k+1} v_{k+2}\right)
$$

and $\beta_{k+1}^{+}$is such that $\left(v_{k+1}^{+}\right)^{T} S v_{k+1}^{+}=1$. Hence equation (14) represents the implicit application of $k$ steps of the Lanczos algorithm to the starting vector $v_{1}^{+}$.

This technique may be iterated. Starting from (14), we repeat one additional step of the Lanczos iteration and then again the implicit method until $\beta_{k+1} \approx 0$.

The application of $p$ shifts $\mu_{1}, \mu_{2}, \ldots, \mu_{p}$ is straightforward and is used to speed-up the computation of $k$ eigenvalues simultaneously. We summarize its use in the following algorithm.

compute $v_{1}, \ldots, v_{k}, \tilde{v}_{k+1}, \alpha_{1}, \ldots, \alpha_{k}, \beta_{2}, \ldots, \beta_{k+1}$ by

means of $k$ steps of the Lanczos algorithm

while $\left|\beta_{k+1}\right|$ is greater than a fixed tolerance

compute $v_{k+1}, \ldots, v_{k+p}, \tilde{v}_{k+p+1}, \alpha_{k+1}, \ldots, \alpha_{k+p}$

$\beta_{k+2}, \ldots, \beta_{k+p+1}$ by means of $p$ additional steps

of the Lanczos algorithm

$v_{k+p+1}=\tilde{v}_{k+p+1} / \beta_{k+p+1}$

construct the matrix $T_{k+p}$ as in (9)

choose the parameters $\mu_{1}, \mu_{2}, \ldots, \mu_{p}$

$Q=I_{k+p} ; \widetilde{T}=T_{k+p}$

for $i=1, \ldots, p$

$Q_{i} R_{i}=\widetilde{T}-\mu_{i} I$

$\widetilde{T}=Q_{i}^{T} \widetilde{T} Q_{i}$

$Q=Q Q_{i}$

end

define $q_{k+p, k}$ as the $(k+p, k)$ element of $Q$

define $\widetilde{\beta}_{k+1}$ as the $(k+1, k)$ element of $\widetilde{T}$

$\widehat{\beta}_{k+p+1}=\beta_{k+p+1} q_{k+p, k}$

$\left[V_{k}^{+} \widehat{V}_{p}\right]=V_{k+p} Q$

define $\hat{v}_{k+1}$ as the first column of $\widehat{V}_{p}$

define $v_{1}, \ldots, v_{k}$ as the $k$ columns of $V_{k}^{+}$

$\tilde{v}_{k+1}=\widetilde{\beta}_{k+1} \hat{v}_{k+1}+\widehat{\beta}_{k+p+1} v_{k+p+1}$

define $\alpha_{1}, \ldots, \alpha_{k}$ and $\beta_{2}, \ldots, \beta_{k}$ as the main diagonal and the lower diagonal of $\widetilde{T}$

$\beta_{k+1}=\left(\tilde{v}_{k+1} S \tilde{v}_{k+1}\right)^{1 / 2}$

end 
The matrix-by-matrix operations are not expensive since $k$ and $p$ are small with respect to $n$. This means that the computational cost of the overall algorithm depends only on the number of iterates in the Lanczos method.

The choice of the parameters $\mu_{j}$ gives rise to different strategies. For example, if we set $\mu_{j}, j=1, \ldots, p$, as $p$ of the eigenvalues of $T_{k+p}$, that is, if we use the exact shift selection strategy [12], then $\widetilde{T}$ has the following structure

$$
\left(\begin{array}{cc}
T_{k}^{+} & \\
& D_{p}
\end{array}\right)
$$

where $D_{p}$ is diagonal with $\mu_{1}, \ldots \mu_{p}$ as main diagonal entries. This strategy gives good results, especially when used to compute the largest eigenvalues in modulus of the positive definite Hamiltonian matrix.

\section{Conclusions}

The Lanczos process has been modified in order to compute the eigenvalues of positive definite Hamiltonian matrices. The obtained algorithm is symplectic and requires half of the workspace of the original algorithm. Moreover, since the procedure gives a symmetric and positive definite matrix, known techniques for this class of matrices (for example, the implicit restarting) can be used to improve the computation.

\section{References}

1. Ammar, G., Mehrmann, V.: On Hamiltonian and symplectic Hessenberg forms. Linear Algebra Appl. 149 (1991), 55-72

2. Amodio, P., Iavernaro, F., Trigiante, D.: Conservative perturbations of positive definite Hamiltonian matrices, Numer. Linear Algebra Appl., (2003), in press

3. Benner, P.: Symplectic balancing of Hamiltonian matrices, SIAM J. Sci. Comput., 22 (5) (2001), 1885-1904

4. Benner, P., Faßbender, H.: An implicit restarted symplectic Lanczos method for the Hamiltonian eigenvalue problem, Linear Algebra Appl., 263 (1997), 75-111

5. Brugnano, L., Trigiante, D.: Solving ODEs by Linear Multistep Initial and Boundary Value Methods. Gordon \& Breach, Amsterdam, 1998

6. Bunse-Gerstner, A.: Matrix factorization for symplectic QR-like methods, Linear Algebra Appl., 83 (1986), 49-77

7. Byers, R.: A Hamiltonian QR-algorithm, SIAM J. Sci. Stat. Comput., 7 (1986), $212-229$

8. Calvetti, D., Reichel, L., Sorensen, D.C.: An implicitly restarted Lanczos method for large symmetric eigenvalue problems, ETNA, Electron. Trans. Numer. Anal., 2 (1994), 1-21

9. Lancaster, P., Rodman, L.: The algebraic Riccati equation. Oxford University Press, Oxford, 1995

10. Lin, W., Mehrmann, V., Xu, H.: Canonical forms for Hamiltonian and symplectic matrices and pencils, Linear Algebra Appl. 302-303 (1999), 469-533 
11. Rosen, I., Wang, C.: A multilevel technique for the approximate solution of operator Lyapunov and algebraic Riccati equations. SIAM J. Matrix Anal. Appl. 32 (1992), $514-541$

12. Sorensen, D.C.: Implicit application of polynomial filters in a $k$-step Arnoldi method, SIAM J. Matrix Anal. Appl. 13 (1992), 357-385

13. Van Loan, C.: A symplectic method for approximating all the eigenvalues of a Hamiltonian matrix, Linear Algebra Appl., 16 (1984), 233-251 